\title{
BMJ Open Helicobacter pylori infection-induced changes in the intestinal microbiota of 14-year-old or 15-year-old Japanese adolescents: a cross-sectional study
}

\author{
Toshihiko Kakiuchi (D , ${ }^{1}$ Yoshiki Tanaka, ${ }^{2}$ Hiroshi Ohno, ${ }^{2}$ Muneaki Matsuo, ${ }^{1}$ \\ Kazuma Fujimoto ${ }^{3}$
}

To cite: Kakiuchi T, Tanaka Y, Ohno $\mathrm{H}$, et al. Helicobacter pylori infection-induced changes in the intestinal microbiota of 14-year-old or 15-year-old Japanese adolescents: a crosssectional study. BMJ Open 2021;11:e047941. doi:10.1136/ bmjopen-2020-047941

- Prepublication history for this paper is available online. To view these files, please visit the journal online (http://dx.doi. org/10.1136/bmjopen-2020047941).

Received 12 December 2020 Accepted 10 June 2021

Check for updates

(c) Author(s) (or their employer(s)) 2021. Re-use permitted under CC BY-NC. No commercial re-use. See rights and permissions. Published by BMJ.

${ }^{1}$ Department of Pediatrics, Saga University, Saga, Japan

${ }^{2}$ R\&D Center, Biofermin Pharmaceutical Co Ltd, Kobe,

Hyogo, Japan

${ }^{3}$ Department of

Gastroenterology, International University of Health and Welfare, Ohkawa, Fukuoka, Japan

Correspondence to

Dr Toshihiko Kakiuchi;

kakiucht@cc.saga-u.ac.jp

\section{ABSTRACT}

Objective The relationship between Helicobacter pylori and the intestinal microbiota has not yet been clearly demonstrated in children and adolescents. The present study aimed at evaluating how $\mathrm{H}$. pylori infection could affect the intestinal microbiota in adolescents using genetic analysis.

Design Cross-sectional study.

Setting and participants We included subjects from a longitudinal project involving $H$. pylori screening and treatment of junior high school third-grade students (aged 14 or 15 years) in Saga Prefecture. The study included a control group $(\mathrm{n}=79)$ and an $H$. pylori group $(\mathrm{n}=80)$ tested negative and positive for the anti-H. pylori antibody in the urine and $H$. pylori antigen in stool specimens, respectively. Interventions The intestinal microbiota was evaluated in stool specimens using 16S rRNA gene/DNA/amplicon sequencing with next-generation sequencing.

Primary and secondary outcome measures We assessed alpha and beta diversity, just as well as relative abundances within the bacterial composition at the genus level in both groups.

Results As shown by the alpha diversity of the 16S rRNA gene/DNA/amplicon sequence data, the control group exhibited lower microbial species richness with lower alpha diversity compared with the $H$. pylori group $(p<0.001)$. The beta diversity of the intestinal microbiota profile also differed between the two groups $(p<0.01)$. The relative abundance of the Prevotella genus was higher in the $H$. pylori group $(p<0.01)$ concomitant with a gain in body mass index $(\mathrm{BMI})$ in the $H$. pylori group $(p<0.01)$ compared with the control group.

Conclusion $H$. pylori infection significantly affected the intestinal microbiota in Japanese adolescents. In addition, the prevalence of the Prevotella genus is concomitantly increased along with the BMI in H. pylori-infected students. Trial registration number UMIN000028721.

\section{INTRODUCTION}

Newborns are exposed to various bacteria that are present in the mother's resident microbiota and the external environment. Bacterial species that comprise the intestinal microbiota change in an age-dependent manner. ${ }^{12}$ The development of the intestinal
Strengths and limitations of this study

- The most significant strength of this study is that it clearly demonstrated the effect of Helicobacter pyIori infection on the intestinal microbiota of children.

- As the participants were Japanese adolescents of almost the same age living in a single prefecture, no major difference would presumably exist between the two groups.

- This study evaluated the intestinal microbiota using faeces specimens, which might differ from the mucosal-associated microbiota.

- The effect of $H$. pylori eradication on the intestinal microbiota could not be analysed, as the eradication therapy is important for intestinal microbiota changes.

microbiota during infancy is affected by several factors, including the maternal resident microbiota, ${ }^{3}{ }^{4}$ the method of nutrition for infants, ${ }^{5-7}$ delivery style ${ }^{589}$ and the administration of antibiotics. $^{3} 1011$

The global Helicobacter pylori prevalence in children varies significantly, from $2.5 \%$ in Japan to $34.6 \%$ in Ethiopia. ${ }^{12}$ Sustained infection of $H$. pylori decreases or increases gastric acid secretion, which might affect the gastric microbiota in adults ${ }^{13-15}$ and children. ${ }^{13} 14$ Several previous reports have suggested that the intestinal microbiota is significantly affected by $H$. pylori infection. ${ }^{15} 16$ The effect of $H$. pylori infection on the intestinal microbiota has been demonstrated in adults, ${ }^{1617}$ but has not been fully investigated in children.

Therefore, the present study aimed at examining junior high school students in Japan aged $14-15$ years to determine whether $H$. pylori infection changes the intestinal microbiota. Moreover, we also examined how body mass index (BMI) affects the intestinal microbiota, in addition to $H$. pylori infection. 
Junior high school third grade students in Saga Prefecture: targeted students for urine H. pylori antibody test in $2017(\mathrm{n}=8,519)$

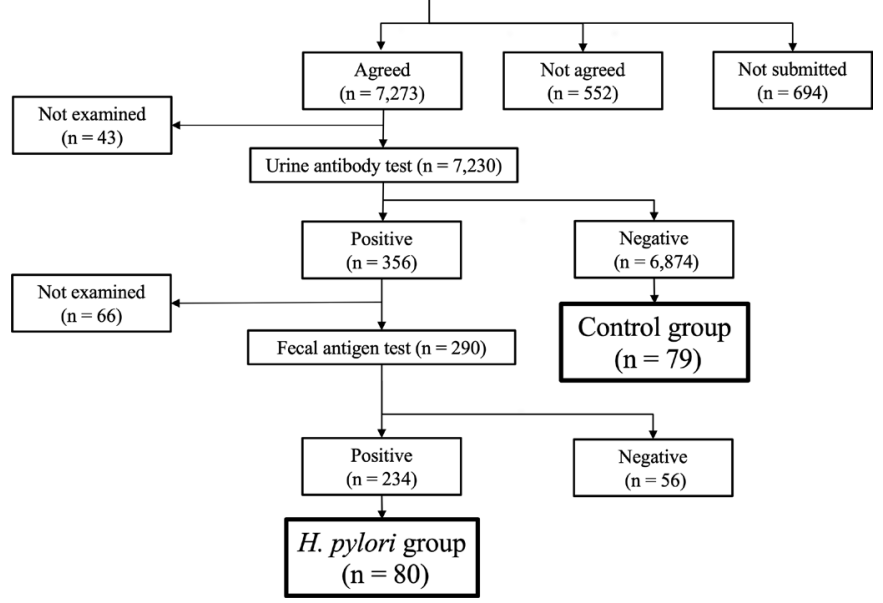

Figure 1 Flowchart for Helicobacter pylori screening and treatment of junior high school students in Saga Prefecture and the selection method used to establish the two groups. The $H$. pylori group comprised 80 students, consented to the study, tested positive for both urinary anti-H. pylori IgG antibody and stool antigen test. The control group $(n=79)$ comprised those tested negative for both tests.

\section{METHODS}

\section{Study design and subjects}

The longitudinal project for $H$. pylori screening and treatment among junior high school third-grade students in Saga Prefecture started in 2016 with the aim of primary prevention of stomach cancer. ${ }^{17}$ Figure 1 shows a flowchart of the junior high school third grade students in Saga Prefecture in 2017. Among 8519 junior high school students aged 14 or 15 years, 7230 received a screening urinary test (RAPIRAN; Otsuka Pharmaceutical Co., Tokyo, Japan) to detect anti-H. pylori IgG antibody by immunochromatography. There is an established screening programme for kidney diseases in Saga Prefecture, targeting third-grade students in junior high schools. Given the full inclusivity of student during this test through simple urine examination, we used the established system to obtain urine samples to screen for H. pylori infection. ${ }^{17}$ The diagnostic sensitivity, specificity, negative predictive value and positive predictive value of the urinary test was reportedly 78.4, 100, 90.1 and $100 \%$, respectively. ${ }^{18}$ A total of 6874 students tested negative for H. pylori with the urinary test and 79 of these students were randomly selected as the $H$. pylori-negative group (control group). Students who tested positive in the screening urinary test received an $H$. pylori stool antigen detection test (Testmate rapid pylori antigen; Wakamoto Pharmaceutical Co., Tokyo, Japan). Among 290 students who received the stool antigen test, 234 students tested positive for $H$. pylori infection. Finally, 80 of these students were randomly selected as the $H$. pylori-positive group $(H$. pylori group). The exclusion criteria for the present study were as follows: (1) students who had taken medications, including proton-pump inhibitors (PPIs), $\mathrm{H}_{2}$ receptor antagonists, antacids, probiotics, mucosal protective agents and antibiotics within the 6 months prior to enrolment, (2) students who were in the outpatient hospital because of sickness, and (3) students who had undergone eradication therapy for $H$. pylori.

The microbiota distribution was compared between the control and $H$. pylori groups regarding alpha diversity, beta diversity and the relative abundance of the intestinal microbiota. The effect of BMI (low: $<15$, middle: 15-25 and high: $>25$ ) on the microbiota distribution in the two groups was examined.

\section{Stool sample collection and bacterial DNA extraction from faeces}

Each participant collected a stool sample at home for the present study using a paper stool collector and tube that was prefilled with $5 \mathrm{~mL}$ of a stool DNA stabiliser. The stool collection method was performed according to the attached document of the stool collection kit. Samples were immediately stored at $-20^{\circ} \mathrm{C}$ and delivered to the project centre within a day. Extraction of bacterial DNA was performed as described previously. ${ }^{19}$ A total of $20 \mathrm{mg}$ of faeces was washed three times in $1.0 \mathrm{~mL}$ of phosphatebuffered saline and centrifuged $(14000 \times g)$. The pellets were resuspended in a solution containing $450 \mu \mathrm{L}$ of extraction buffer (100 mM Tris-HCl, 40 mM EDTA; $\mathrm{pH}$ 9.0) and $50 \mu \mathrm{L}$ of $10 \%$ sodium dodecyl sulfate. A total of $300 \mathrm{mg}$ of glass beads (diameter, $0.1 \mathrm{~mm}$ ) and $500 \mu \mathrm{L}$ of buffer-saturated phenol were added to the suspension and vortexed vigorously. After centrifugation at $14000 \times$ $g$ for $5 \mathrm{~min}, 400 \mu \mathrm{L}$ of the supernatant was extracted by phenol-chloroform, and $250 \mu \mathrm{L}$ of the supernatant was subjected to isopropanol precipitation. Finally, the DNA was suspended in $1.0 \mathrm{~mL}$ of Tris-EDTA buffer.

\section{DNA sequence analysis}

We performed the meta-analysis of the bacterial 16S rDNA sequences in the faeces in accordance with a previously described method ${ }^{20}$ with minor modifications. Briefly, the V3-V4 region of $16 \mathrm{~S}$ rDNA were amplified on a Veriti thermal cycler (Thermo Fisher Scientific, Waltham, Massachusetts, USA). The amplicon was purified using AMPure XP magnetic beads (Beckman Coulter, Brea, California, USA). For multiplex sequencing, a PCR was performed with dual eight-base indices (Nextera XT Index kit, Illumina, California, USA). After purification by AMPure XP beads, the purified barcoded library was quantified fluorometrically using a QuantiT PicoGreen ds DNA Assay Kit (Invitrogen, Paisley, UK) and pooled at the same volume. The library pool $(10 \mathrm{pM})$ was spiked with $40 \%$ PhiX control DNA (10 pM). Sequencing was conducted on a MiSeq platform with MiSeq Reagent Kit v2 chemistry (Illumina).

\section{Microbiota analysis}

We conducted the removal of low-quality and chimaera sequences, construction of operational taxonomic units (OTUs) and taxonomy assignment using the Quantitative 
Insights Into Microbial Ecology pipeline (http://qiime. org/).${ }^{21}$ Briefly, 50000 raw reads were randomly obtained from the sequence files for each sample and merged by fastq-join with the default setting. Consequently, sequence reads with an average quality value of $<25$ were removed and then chimera-checked. Five thousand reliable sequence reads were randomly obtained for each sample and OTUs were constructed by clustering with a 97\% identity threshold. The representative reads of each OTU were then assigned to the $16 \mathrm{~S}$ rRNA gene database using UCLUST with $\geq 97 \%$ identity. ${ }^{22}$ A comparison of each taxon in the gut microbiota was conducted at the genus level. Beta diversity was estimated by computing the weighted and unweighted UniFrac distances between the samples. ${ }^{23}$ In order to compare the differences in the overall bacterial gut microbiota structure, principal coordinates analysis was applied to reduce the dimensionality of the resulting distance matrix. We calculated the Shannon index, observed OTUs, chao 1, and the abundance-based coverage estimator (ACE) index to investigate the alpha diversity of the microbiota in the samples.

\section{Statistical analysis}

All statistical analyses were conducted with the $\mathrm{R}$ statistical software (R Core Team (2018); R: A language and environment for statistical computing; $\mathrm{R}$ Foundation for Statistical Computing, Vienna, Austria; https://www. R-project.org/). Data are shown as the mean \pm SE. Statistical significance was set at $\mathrm{p}<0.05$. During the analyses of the gut microbiotas, the statistical significance was determined by Welch's t-test with Benjamini-Hochberg correlation. The relative abundance data were nonnormally distributed. However, we applied Welch's t-test as the Mann-Whitney U-test is reportedly less robust. ${ }^{24}$ Beta diversity was analysed using permutational analysis of multivariate dispersions (PERMDISP) for comparisons of gene similarity.

\section{Patient and public involvement}

This study was performed without patient involvement. Patients were not invited to comment on the study design and were not consulted to develop patientrelevant outcomes or interpret the results. Patients were not invited to contribute to the writing or editing of the manuscript for readability or accuracy.

\section{RESULTS}

\section{Student characteristics}

A total of 159 students participated in this study. The student characteristics are shown in table 1. No significant differences could be observed in sex, age, BMI, birth delivery style, method of infant nutrition or the prevalence of allergic disease between the groups. The ratio of nursery school graduates to kindergarten graduates was significantly higher in the $H$. pylori group than in the control group $(\mathrm{p}<0.001)$. The subjects of this study did not include low-birth-weight infants (birth weight $2500 \mathrm{~g}$ or less). In addition, we did not investigate whether symptoms associated with $H$. pylori infection, such as abdominal symptoms, were present in the H. pylori group.

\section{Alpha and beta diversity in the control and the $H$. pylori groups}

Figure 2 shows the alpha diversity of the 16S rRNA gene/DNA/amplicon sequence data. The control group showed lower microbial species richness with lower alpha diversity compared with the $H$. pylori group. The observed species index, chao 1 index, and ACE index all showed significantly higher diversity in the $H$. pylori group compared with the control group $(\mathrm{p}<0.001)$. The Shannon index was not significantly different between the two groups $(\mathrm{p}=0.054)$.

Figure 3 shows the beta diversity of the $16 \mathrm{~S}$ rRNA gene/ DNA/amplicon sequence data. The two-dimensional principal coordinate analysis of the weighted and unweighted UniFrac distances of the 16S rRNA gene/DNA/amplicon sequence data showed that the majority of samples were clustered dependent on the $H$. pylori infection status. The similarity analysis showed that the differences were significant for the weighted UniFrac distance $(p<0.001)$, but not for the unweighted UniFrac distance $(\mathrm{p}=0.643)$ using PERMDISP.

Table 1 Background characteristics of junior high school students in the two groups

\begin{tabular}{llll}
\hline & Control group $(\mathbf{n}=\mathbf{7 9})$ & Helicobacter pylori group $(\mathbf{n = 8 0})$ & P value \\
\hline Sex (male/female) & $42 / 37$ & $46 / 34$ & 0.80 \\
Age (years) & $14.73 \pm 0.33$ & $14.76 \pm 0.32$ & 0.71 \\
Body mass index $\left(\mathrm{kg} / \mathrm{m}^{2}\right)$ & $19.69 \pm 3.48$ & $19.67 \pm 2.41$ & 0.97 \\
Delivery (vaginal/caesarean section) & $68 / 11$ & $60 / 11$ & 0.79 \\
Nutrition (breast/formula/mix) & $37 / 6 / 36$ & $27 / 15 / 36$ & 0.07 \\
School (nursery/kindergarten/none) & $25 / 54 / 0$ & $53 / 25 / 2$ & $<0.001$ \\
Allergies (+/-) & $5 / 75$ & $7 / 73$ & 0.55 \\
\hline
\end{tabular}

Delivery, birth delivery style; nutrition, method of infant nutrition; school, pre-school situation. 

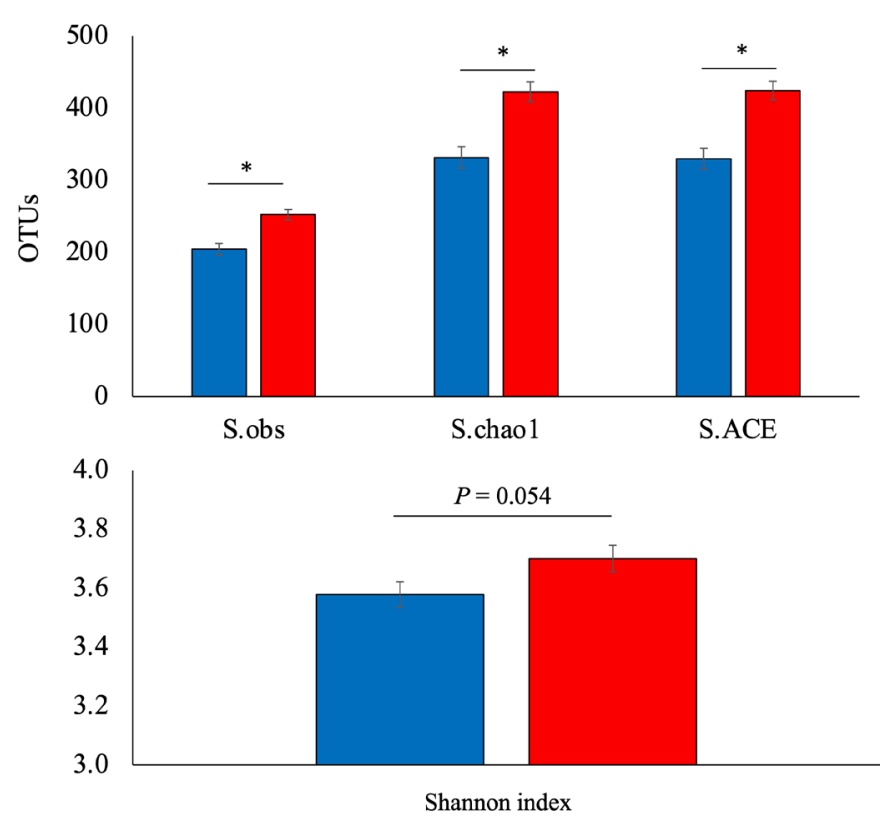

Control group

H. pylori group

Figure 2 Alpha diversity of the 16S rRNA sequences in the control and Helicobacter pylori groups The control group exhibited lower microbial species richness compared with the $H$. pylori group. The S.obs index, chao 1 index and ACE index, all showed significantly higher diversity in the $H$. pylori group than in the control group $\left({ }^{*} p<0.001\right)$. The Shannon index was not significantly different between the two groups $(p=0.054)$. ACE, abundance-based coverage estimator; obs, observed; OTU, operational taxonomic unit; S, species.

\section{Relative abundances within the bacterial composition at the genus level for the two groups}

Figure 4 shows the 13 main bacterial types present in the intestinal microbiota at the genus level as follows: Bacteroides, Blautia, Bifidobacterium, Faecalibacterium, Prevotella, Fusicatenibacter, Eubacterium, Anaerostipes, Subdoligranulum, Streptococcus, Megamonas, Collinsella and Clostridium. The relative abundances of the Prevotella $(\mathrm{p}<0.01)$ and
A: Weighted UniFrac distance

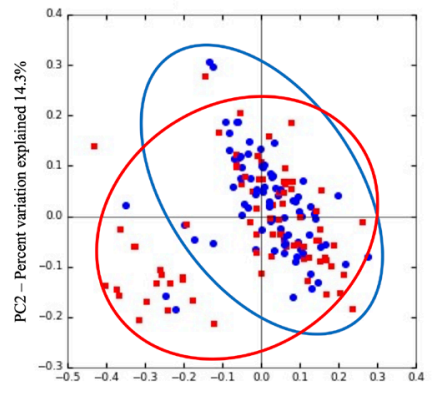

PC1 - Percent variation explained 28.3\%

(PERMDISP $P<0.001$ )

Control group
B: Unweighted UniFrac distance

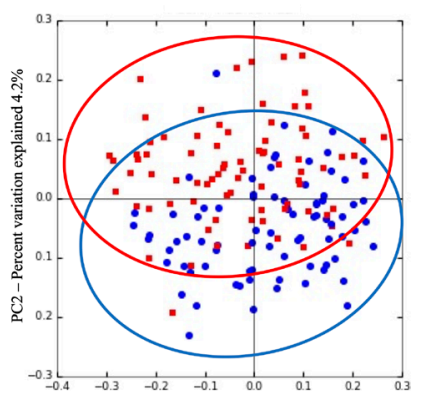

$\mathrm{PCl}$ - Percent variation explained $8.8 \%$ (PERMDISP $P<0.643$ )

H. pylori group
Figure 3 Beta diversity of the 16S rRNA/DNA/amplicon sequence data (control group vs Helicobacter pylori group). PC, principal coordinate; PERMDISP, permutational analysis of multivariate dispersions.

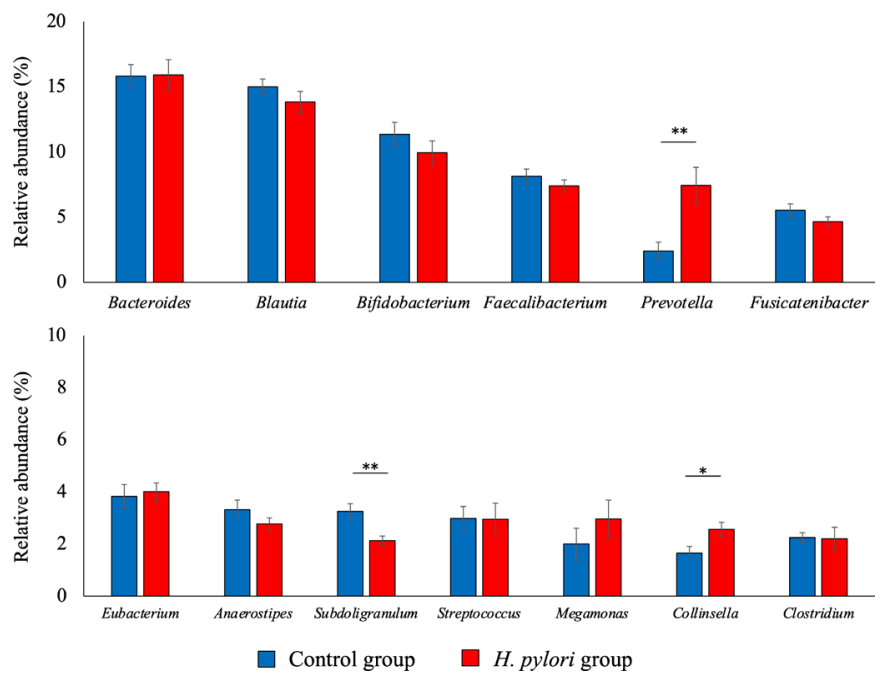

Figure 4 The main 13 bacterial types present in the intestinal microbiota at the genus level, comparing the Helicobacter pylori and control groups $\left({ }^{\star} \mathrm{p}<0.05\right.$; $\left.{ }^{\star \star} \mathrm{p}<0.01\right)$.

Collinsella genus $(\mathrm{p}<0.05)$ were significantly higher in the $H$. pylori group than in the control group. The relative abundance of the Subdoligranulum genus was significantly higher in the control group than in the H. pylori group $(p<0.01)$. At the phylum level, the ratio of the Firmicutes to the Bacteroides phyla (F/B ratio) showed no significant difference between the two groups (the control group $4.19 \pm 3.27$ vs the $H$. pylori group $4.87 \pm 12.04$, $\mathrm{p}=0.63$ ).

$\mathrm{BMI}$ and the relative abundances within the bacterial composition at the genus level

In the control and $H$. pylori groups, the intestinal microbiota was evaluated in association with the BMI. Figure 5 shows the seven main bacterial types in the intestinal microbiota at the genus level for the control and the $H$. pylori groups, categorised by the BMI. For the control group, these included Bacteroides, Blautia, Bifidobacterium, Prevotella, Faecalibacterium, Fusicatenibacter and Megamonas and for the H. pylori group Prevotella, Bacteroides, Blautia, Bifidobacterium, Faecalibacterium, Megamonas and Fusicatenibacter. In the H. pylori group, the relative abundance of the Prevotella genus was significantly higher in the high BMI group compared with the middle and low BMI groups (both $\mathrm{p}<0.01$ ). Furthermore, the relative abundance of the Prevotella genus in the middle BMI group was higher than that in the low BMI group $(\mathrm{p}<0.05)$. The relative abundances of Bacteroides and Bifidobacterium were significantly lower in the high BMI group compared with the other two groups (both $\mathrm{p}<0.05$ ). In the $H$. pylori group, the BMI did not affect the relative abundances of Blautia, Faecalibacterium, Magamonas and Fusicatenibacter. In the control group, the relative abundance of the Prevotella genus was not significantly higher in the high BMI group compared with the middle and low BMI groups, whereas the relative abundance of the Prevotella genus significantly and proportionately increased with an increasing BMI in the $H$. pylori group (low BMI vs high BMI: $\mathrm{p}<0.001$, middle BMI vs high BMI: $\mathrm{p}<0.001$ ) (figure 6 ). At the 


\section{(A)}

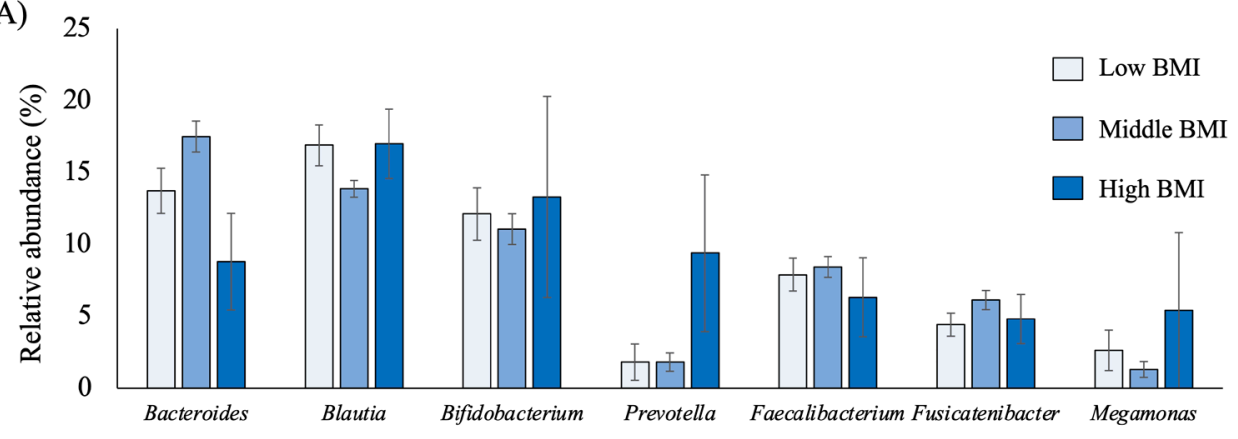

(B)

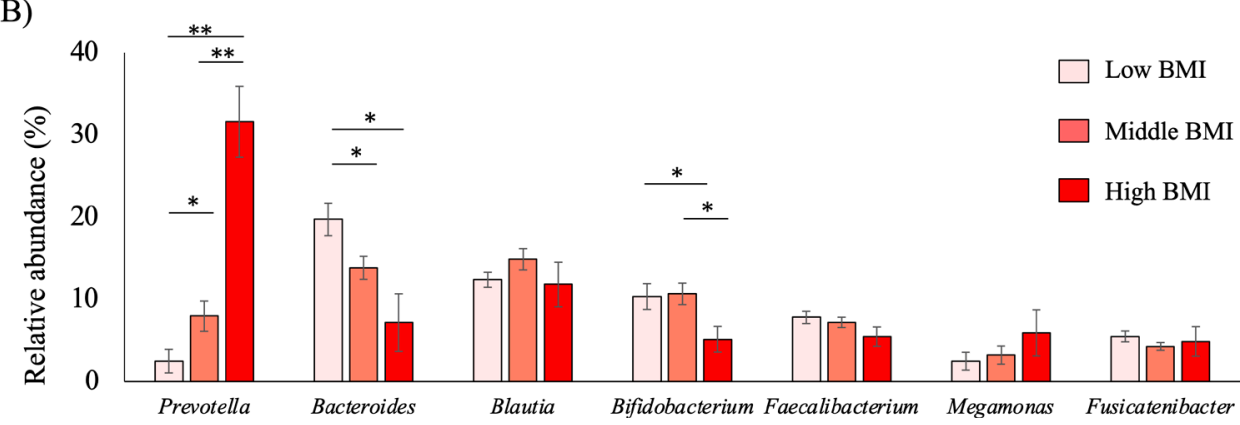

Figure 5 The seven main bacterial types present in the intestinal microbiota at the genus level for the control group (A) and the Helicobacter pylori group (B) in association with body mass index (BMI) ( ${ }^{*} \mathrm{p}<0.05$; $\left.{ }^{* \star} \mathrm{p}<0.01\right)$. Low: $\mathrm{BMI}<15 \mathrm{~kg} / \mathrm{m}^{2}$; middle: BMI $15-25 \mathrm{~kg} / \mathrm{m}^{2}$; high: BMl $>25 \mathrm{~kg} / \mathrm{m}^{2}$.

phylum level, we observed no significant differences in the $\mathrm{F} / \mathrm{B}$ ratio among the three $\mathrm{BMI}$ categories in the control groups. However, a significant difference could be detected between the high and middle BMI categories in the $H$. pylori group (figure 7 ). The Subdoligranulum genus had a lower relative abundance in the high BMI category than in the low BMI group, although this trend was observed not only in the $H$. pylori group, but also in the control group (figure 8). The Collinsella genus was not associated with the BMI regardless of $H$. pylori infection status (figure 9).

\section{DISCUSSION}

The present study revealed two clinically important results: (1) $\mathrm{H}$. pylori infection significantly affected the intestinal microbiota of adolescents aged 14 or 15 years, as determined for Japanese junior high school students; (2) an increase in the relative abundance of the Prevotella genus in $H$. pylori-infected adolescents was concomitant with a gain in BMI.

Most reports of the effects of $H$. pylori on the intestinal microbiota based on the analysis of faeces samples were in adults and data were lacking for children. ${ }^{25} 26$ The present study showed a difference in the intestinal microbiota between $H$. pylori-infected and non-infected adolescents based on faeces specimens. Alpha diversity, bacterial richness and variance all showed greater diversity in $H$. pylori-infected students than in controls (figure 2). A previous study showed that the diversity of the gastric microbiota in adolescents was enhanced by $H$. pylori infection. ${ }^{13}$ Studies of the relationship between the intestinal microbiota and $H$. pylori infection are limited. One study reported a decrease in the Firmicutes genus in the human duodenal mucosa during $H$. pylori infection. ${ }^{27}$ In the $H$. pylori infection model of Mongolian gerbils, the abundances of the Bacteroides and Enterococcus genera were increased in the duodenal mucosa. ${ }^{28}$ In adults, $H$. pylori infection ${ }^{17}$ reportedly reduced intestinal microbiota diversity and our results were in good agreement with these previous reports (figures 2 and 3 ). The human gut microbiota has been reported to form by the age of 3 years, ${ }^{29}$ so it may be that there is no difference in the effects of $H$. pylori infection on the intestinal microbiota between adolescents and adults.

It is known that infection with $H$. pylori reduces gastric acid secretion in children. ${ }^{30} 31$ It was further suggested that a decrease in gastric acid secretion due to $H$. pylori infection may affect the intestinal flora of adolescents with $H$. pylori infection. In addition, a decrease in gastric acid secretion caused by $H$. pylori infection may allow a wide variety of bacteria in the oral cavity to more easily pass through the stomach and reach the lower gastrointestinal tract, thereby affecting the intestinal flora in faeces. The inhibitory effect of PPIs on gastric acid secretion affects the composition of the intestinal flora. Administration of PPIs causes an increase in the indigenous bacteria, the Streptococcus and Lactobacillus genus in the intestine, which is thought to be due to the oral bacteria reaching the intestine to suppress gastric acid secretion. ${ }^{32}{ }^{33}$ This might explain the result of the present study that alpha 


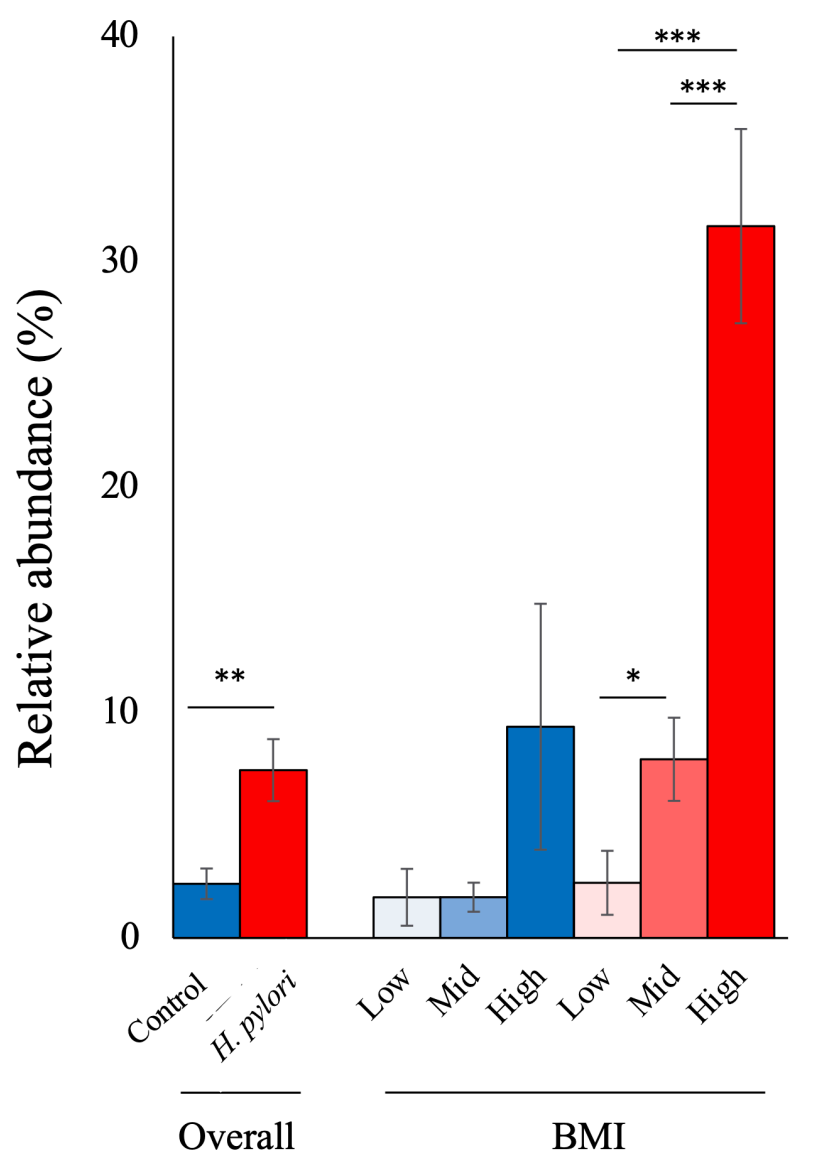

\section{Control group $\square$ H.pylori group}

Figure 6 Relative abundance of the Prevotella genus in relation to the body mass index (BMI) category in the Helicobacter pylori and control groups $\left({ }^{\star} \mathrm{p}<0.05 ;{ }^{* \star} \mathrm{p}<0.01\right.$; ${ }^{* \star *} \mathrm{p}<0.001$ ). Low: $\mathrm{BMl}<15 \mathrm{~kg} / \mathrm{m}^{2}$; mid BMl $15-25 \mathrm{~kg} / \mathrm{m}^{2}$; high $\mathrm{BMl}>25 \mathrm{~kg} / \mathrm{m}^{2}$.
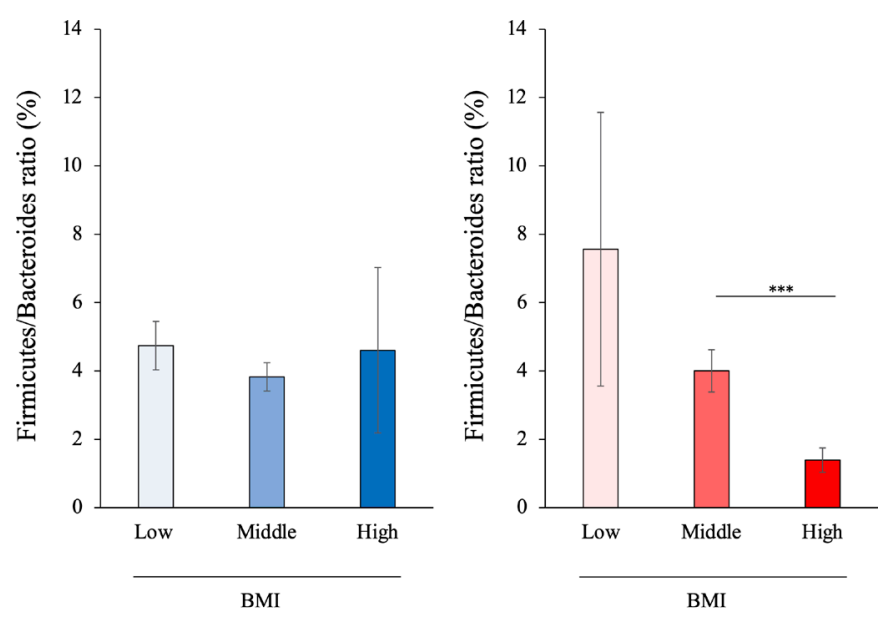

Figure 7 The ratio of the Firmicutes phylum to the Bacteroides phylum in relation to the body mass index (BMI) category in the Helicobacter pylori and control groups $\left({ }^{\star \star \star} \mathrm{p}<0.001\right)$. Low: $\mathrm{BMl}<15 \mathrm{~kg} / \mathrm{m}^{2}$; middle: BMl $15-25 \mathrm{~kg} / \mathrm{m}^{2}$; high: $\mathrm{BMl}>25 \mathrm{~kg} / \mathrm{m}^{2}$.

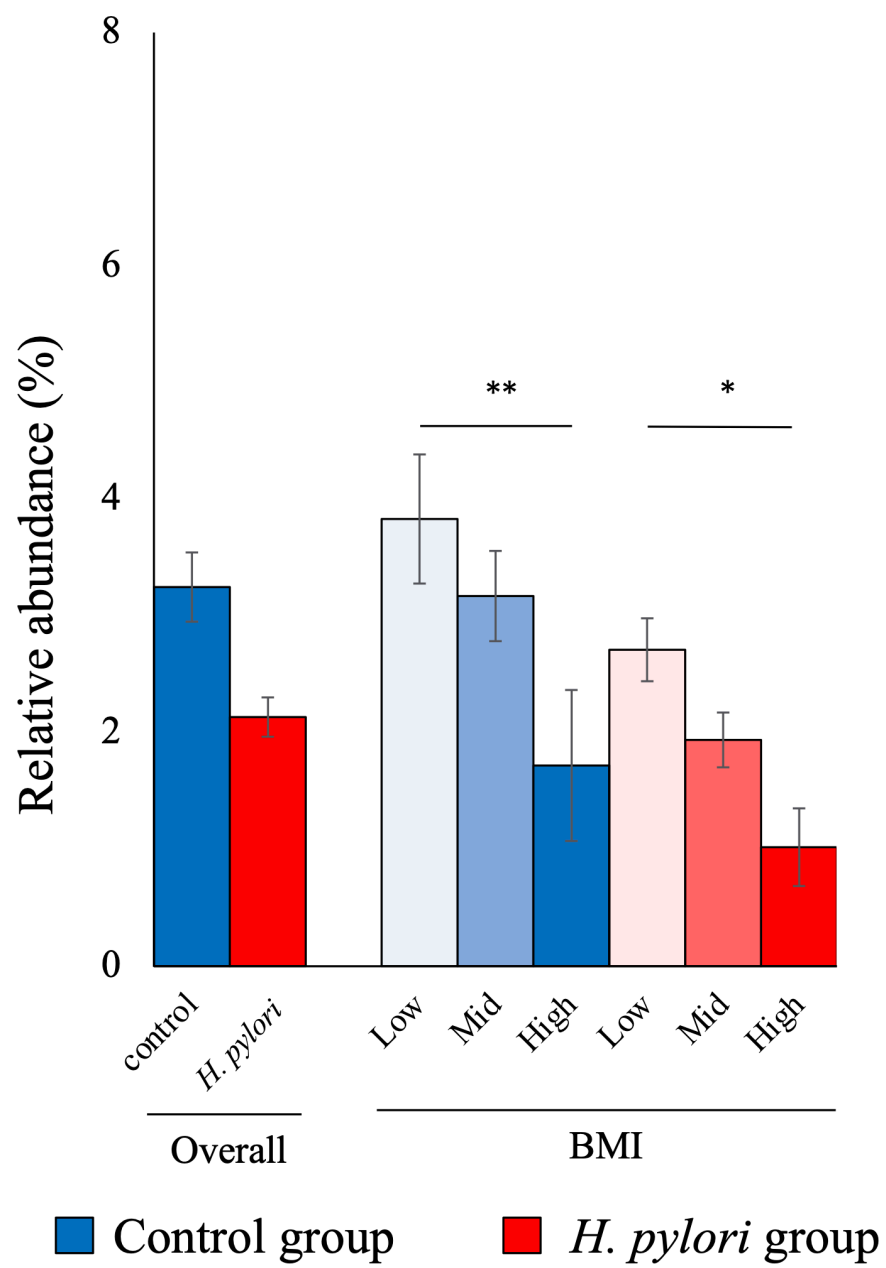

Figure 8 Relative abundance of the Subdoligranulum genus in relation to the body mass index (BMI) category in the Helicobacter pylori and control groups $\left({ }^{*} \mathrm{p}<0.05 ;{ }^{* *} \mathrm{p}<0.01\right)$. Low: $\mathrm{BMI}<15 \mathrm{~kg} / \mathrm{m}^{2}$; mid: BMI $15-25 \mathrm{~kg} / \mathrm{m}^{2}$; high: $\mathrm{BMI}>25$ $\mathrm{kg} / \mathrm{m}^{2}$.

diversity of the faecal intestinal microbiota was increased in students with $H$. pylori infection. As suggested by the present study, $H$. pylori infection might be a factor that disturbs the intestinal microbiota in adolescents. H. pylori infection is involved in the alterations of gut microbiota composition and diversity, which can lead to changes in production level and physiological regulation of the gut metabolic hormones released from the host endocrine system. ${ }^{34}$ The mechanisms and clinical importance of the effect of $H$. pylori warrant further investigation.

The Prevotella genus increased in abundance during $H$. pylori infection, and this increase was found to be concomitant with a rise in BMI in the present study. A previous report indicated that the Prevotella genus was elevated in abundance in school-age children infected with $H$. pylori. ${ }^{35}$ This was an epidemiological study, and unfortunately, it is completely unknown why at this time the Prevotella genus is elevated in school-age children infected with $H$. pylori. The Bacteroides and Bifidobacterium genera are dominant among the intestinal microbiota in Japanese children. ${ }^{36}$ A previous study showed that the prevalence rate of the Prevotella genus in the intestinal microbiota was higher in 


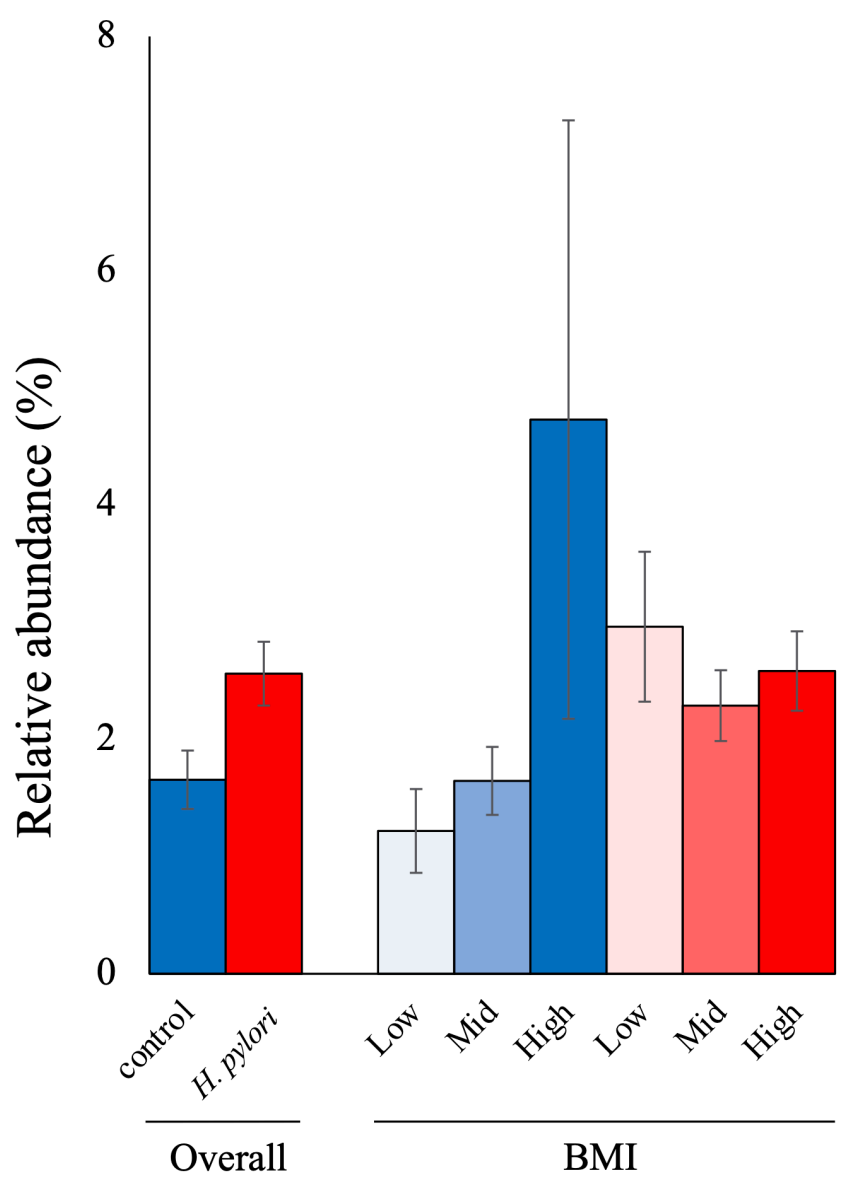

$\square$ Control group $\square$ H.pylori group

Figure 9 Relative abundance of the Collinsella genus in relation to the body mass index (BMI) category in the Helicobacter pylori and control groups. Low: BMI $<15 \mathrm{~kg} / \mathrm{m}^{2}$; mid: BMI 15-25 kg/m²; high: BMI >25 kg/m².

subjects who consumed carbohydrates more frequently, ${ }^{37}$ which suggests that the Prevotella genus is closely related to eating habits. In the present study, it is not possible to determine whether infection with $H$. pylori affected the diet and resulted in an increase in the Prevotella genus, or whether infection with $H$. pylori increased the Prevotella genus and affected the diet, and caused an increase in BMI. In general, the F/B ratio has been found to increase with obesity. ${ }^{38}$ The Prevotella genus belongs to the Bacteroides phylum, the present study thus exhibited inconsistency. At the moment, it is currently difficult to associate the $\mathrm{F} / \mathrm{B}$ ratio with a determined health status and, more specifically, to consider it as a hallmark of obesity. ${ }^{39}$ In the future, regarding the relationship between $H$. pylori and the Prevotella genus and BMI, it is necessary to analyse the intestinal flora in early childhood, including the history of eating habits from early childhood.

A correlation between $H$. pylori infection and the onset of diabetes has been reported in epidemiology studies, ${ }^{4041}$ but the reason for this remains unknown. Meanwhile, the prevalence of the Prevotella genus increased in patients with obesity, ${ }^{42}{ }^{43}$ non-alcoholic steatohepatitis, ${ }^{44}$ hyperlipidaemia, ${ }^{45}$ and even in gestational diabetes, which is considered as a diabetes mellitus preliminary group. ${ }^{46}$ The Prevotella genus is considered to contribute to hyperglycaemia and insulin resistance. ${ }^{43} 4788$ In the present study, an increase in the relative abundance of the Prevotella genus was observed in $H$. pylori-infected children with an increased BMI (figures 5 and 6). $H$. pylori infection in children with an elevated BMI without diabetes mellitus, caused an increase in the prevalence of the Prevotella genus (figures 5 and 6) and, as a result, insulin resistance increased, which may predispose individuals to diabetes mellitus. In fact, it is thought that the increase in Prevotella genus may be involved in the process of developing abnormal glucose metabolism as a result of obesity. ${ }^{450}$

The Subdoligranulum genus showed a lower relative abundance in the high BMI category than in the low BMI group, but this trend was seen not only in the $H$. pylori group, but also in the control group (figure 7). The Collinsella genus was not associated with BMI regardless of H. pylori infection status (figure 8). It has been reported that the Subdoligranulum genus is less prevalent among type 2 diabetes patients compared with their non-diabetic counterparts, ${ }^{51}$ and a negative correlation with insulin resistance has been shown. ${ }^{52}$ An increase in the Collinsella genus is reportedly associated with increased insulin, triglyceride and very-low-density lipoprotein levels, ${ }^{53}$ and is associated with type 2 diabetes. ${ }^{54}$ In our study, of the three genera (Prevotella, Subdoligranylum and Collinsella) that showed significant differences in relative abundance between the H. pylori and control groups, the Prevotella genus showed the most significant correlation between H. pylori infection status and BMI. The Prevotella genus was the only genus that showed an association with BMI in the H. pylori group, but not the control group.

There are several limitations to the present study. (1) In the selection of subjects in both groups, false negative results by using the urinary antibody in the control group and false positive results by using stool antigen in the $H$. pylori group could not be completely eliminated. (2) The present study evaluated faeces specimens, the microbiota of which may be different from the mucosal-associated microbiota. (3) The effect of eradication of $H$. pylori on the intestinal microbiota could be important, ${ }^{55}$ and we plan to investigate this in the future. (4) There was a difference in preschool status between the two groups (table 1), and it could not be completely ruled out that this could have affected the intestinal microbiota.

\section{CONCLUSION}

The present study shows that the intestinal microbiota is significantly affected by $H$. pylori infection in junior high school third grade students in Saga Prefecture, Japan. Furthermore, the relative abundance of the Prevotella genus was increased concomitantly with a rise in BMI in H. pylori-infected students. 
Acknowledgements We would like to thank Mr Daisuke Takami of R\&D Centre, Biofermin Pharmaceutical Co, Ltd, for his cooperation. We thank Ms Kozue Kakiuchi, Ms Tomomi Ito and Ms Hiromi Beppu for project support.

Contributors This study was supported by the Biofermin Pharmaceutical Co, Ltd, (Kobe, Japan), performing intestinal microbiota analysis and statistical evaluation. However, their contribution did not influence data analysis or interpretation in this study. The authors (YT and $\mathrm{HO}$ ) did not play any additional role in the study design, data collection and analysis, publishing decisions or manuscript preparation. Study concept and design: TK and KF. Acquisition of data: TK. Analysis and interpretation of data: TK. Drafting of the manuscript: TK. Critical revision of the manuscript for important intellectual content: MM and KF. Statistical analysis: YT and HO. Administrative, technical or material support: YT and HO. Study supervision: MM and KF. Writing, reviewing and editing: MM and KF.

Funding The authors have not declared a specific grant for this research from any funding agency in the public, commercial or not-for-profit sectors.

Competing interests None declared.

Patient and public involvement Patients and/or the public were not involved in the design, or conduct, or reporting, or dissemination plans of this research.

Patient consent for publication Not required.

Ethics approval The ethical aspects of this study were reviewed and approved by the institutional review board of Saga University Hospital (approval number: 201611-03). Written informed consent was obtained from all of the students and their guardians. All methods were carried out in accordance with relevant guidelines and regulations or Helsinki guidelines.

Provenance and peer review Not commissioned; externally peer-reviewed.

Data availability statement Data are available upon reasonable request. The data sets used and/or analysed during the current study are available from the corresponding author on reasonable request.

Open access This is an open access article distributed in accordance with the Creative Commons Attribution Non Commercial (CC BY-NC 4.0) license, which permits others to distribute, remix, adapt, build upon this work non-commercially, and license their derivative works on different terms, provided the original work is properly cited, appropriate credit is given, any changes made indicated, and the use is non-commercial. See: http://creativecommons.org/licenses/by-nc/4.0/.

ORCID iD

Toshihiko Kakiuchi http://orcid.org/0000-0002-9995-5522

\section{REFERENCES}

1 Matsuki T, Yahagi K, Mori $\mathrm{H}$, et al. A key genetic factor for fucosyllactose utilization affects infant gut microbiota development. Nat Commun 2016;7:11939.

2 Yatsunenko T, Rey FE, Manary MJ, et al. Human gut microbiome viewed across age and geography. Nature 2012;486:222-7.

3 Romero R, Hassan SS, Gajer P, et al. The composition and stability of the vaginal microbiota of normal pregnant women is different from that of non-pregnant women. Microbiome 2014;2:4.

4 Aagaard K, Riehle K, Ma J, et al. A metagenomic approach to characterization of the vaginal microbiome signature in pregnancy. PLoS One 2012;7:e36466.

5 Bäckhed F, Roswall J, Peng Y, et al. Dynamics and stabilization of the human gut microbiome during the first year of life. Cell Host Microbe 2015;17:690-703.

6 Bezirtzoglou E, Tsiotsias A, Welling GW. Microbiota profile in feces of breast- and formula-fed newborns by using fluorescence in situ hybridization (fish). Anaerobe 2011;17:478-82.

7 Penders J, Vink C, Driessen C, et al. Quantification of Bifidobacterium spp., Escherichia coli and Clostridium difficile in faecal samples of breast-fed and formula-fed infants by real-time PCR. FEMS Microbiol Lett 2005;243:141-7.

8 Bokulich NA, Chung J, Battaglia T, et al. Antibiotics, birth mode, and diet shape microbiome maturation during early life. Sci Transl Med 2016;8:343ra82.

9 Mueller NT, Bakacs E, Combellick J, et al. The infant microbiome development: mom matters. Trends Mol Med 2015;21:109-17.

10 Fouhy F, Guinane CM, Hussey S, et al. High-Throughput sequencing reveals the incomplete, short-term recovery of infant gut microbiota following parenteral antibiotic treatment with ampicillin and gentamicin. Antimicrob Agents Chemother 2012;56:5811-20.
11 Tanaka S, Kobayashi T, Songjinda P, et al. Influence of antibiotic exposure in the early postnatal period on the development of intestinal microbiota. FEMS Immunol Med Microbiol 2009;56:80-7.

12 Mišak Z, Hojsak I, Homan M. Review: Helicobacter pylori in pediatrics. Helicobacter 2019;24 Suppl 1:e12639.

13 Brawner KM, Kumar R, Serrano CA, et al. Helicobacter pylori infection is associated with an altered gastric microbiota in children. Mucosal Immunol 2017;10:1169-77.

14 Llorca L, Pérez-Pérez G, Urruzuno P, et al. Characterization of the gastric microbiota in a pediatric population according to Helicobacter pylori status. Pediatr Infect Dis J 2017;36:173-8.

15 Oh B, Kim B-S, Kim JW, et al. The effect of probiotics on gut microbiota during the Helicobacter pylori eradication: randomized controlled trial. Helicobacter 2016;21:165-74.

16 Bühling A, Radun D, Müller WA, et al. Influence of anti-Helicobacter triple-therapy with metronidazole, omeprazole and clarithromycin on intestinal microflora. Aliment Pharmacol Ther 2001;15:1445-52.

17 Kakiuchi T, Matsuo M, Endo $\mathrm{H}$, et al. A Helicobacter pylori screening and treatment program to eliminate gastric cancer among junior high school students in SAGA Prefecture: a preliminary report. J Gastroenterol 2019;54:699-707

18 Okuda M, Kamiya S, Booka M, et al. Diagnostic accuracy of urinebased kits for detection of Helicobacter pylori antibody in children. Pediatr Int 2013;55:337-41.

19 Matsuki T, Watanabe K, Fujimoto J, et al. Quantitative PCR with 16S rRNA-gene-targeted species-specific primers for analysis of human intestinal bifidobacteria. Appl Environ Microbiol 2004;70:167-73.

20 Fadrosh DW, Ma B, Gajer P, et al. An improved dual-indexing approach for multiplexed 16S rRNA gene sequencing on the Illumina MiSeq platform. Microbiome 2014;2:6.

21 Caporaso JG, Kuczynski J, Stombaugh J, et al. QIIME allows analysis of high-throughput community sequencing data. Nat Methods 2010;7:335-6.

22 Edgar RC. Search and clustering orders of magnitude faster than blast. Bioinformatics 2010;26:2460-1.

23 Lozupone C, Knight R. UniFrac: a new phylogenetic method for comparing microbial communities. Appl Environ Microbiol 2005;71:8228-35.

24 Asaka M. A new approach for elimination of gastric cancer deaths in Japan. Int J Cancer 2013;132:1272-6.

25 Dash NR, Khoder G, Nada AM, et al. Exploring the impact of Helicobacter pylori on gut microbiome composition. PLoS One 2019;14:e0218274.

26 Yang Y-J, Sheu B-S. Metabolic interaction of Helicobacter pylori infection and gut microbiota. Microorganisms 2016;4. doi:10.3390/ microorganisms4010015. [Epub ahead of print: 1602 2016].

27 Schulz C, Schütte K, Koch N, et al. The active bacterial assemblages of the upper GI tract in individuals with and without Helicobacter infection. Gut 2018;67:216-25.

28 Yin Y-N, Wang C-L, Liu X-W, et al. Gastric and duodenum microflora analysis after long-term Helicobacter pylori infection in Mongolian gerbils. Helicobacter 2011;16:389-97.

29 Mitsuoka T, Hayakawa K, Kimura N. [The faecal flora of man. II. The composition of bifidobacterium flora of different age groups (author's transl)]. Zentralbl Bakteriol Orig A 1974;226:469-78.

30 Boukthir S, Aouididi F, Mazigh Mrad S, et al. [Chronic gastritis in children]. Tunis Med 2007;85:756-60.

31 Yu Y, Su L, Wang X, et al. Association between Helicobacter pylori infection and pathological changes in the gastric mucosa in Chinese children. Intern Med 2014;53:83-8.

32 Hojo M, Asahara T, Nagahara A, et al. Gut microbiota composition before and after use of proton pump inhibitors. Dig Dis Sci 2018;63:2940-9.

33 Kinoshita Y, Ishimura N, Ishihara S. Advantages and disadvantages of long-term proton pump inhibitor use. J Neurogastroenterol Motil 2018;24:182-96.

34 Mohammadi SO, Yadegar A, Kargar M, et al. The impact of Helicobacter pylori infection on gut microbiota-endocrine system axis; modulation of metabolic hormone levels and energy homeostasis. J Diabetes Metab Disord 2020;19:1855-61.

35 Benavides-Ward A, Vasquez-Achaya F, Silva-Caso W, et al. Helicobacter pylori and its relationship with variations of gut microbiota in asymptomatic children between 6 and 12 years. BMC Res Notes 2018;11:468.

36 Nakayama J, Watanabe K, Jiang J, et al. Diversity in gut bacterial community of school-age children in Asia. Sci Rep 2015;5:8397.

37 Lim MY, Rho M, Song Y-M, et al. Stability of gut enterotypes in Korean monozygotic twins and their association with biomarkers and diet. Sci Rep 2014;4:7348.

38 Ley RE, Turnbaugh PJ, Klein S, et al. Microbial ecology: human gut microbes associated with obesity. Nature 2006;444:1022-3. 
39 Magne F, Gotteland M, Gauthier L, et al. The Firmicutes/ Bacteroidetes ratio: a relevant marker of gut dysbiosis in obese patients? Nutrients 2020;12. doi:10.3390/nu12051474. [Epub ahead of print: 19 May 2020].

40 Jeon CY, Haan MN, Cheng C, et al. Helicobacter pylori infection is associated with an increased rate of diabetes. Diabetes Care 2012;35:520-5.

41 Marietti M, Gasbarrini A, Saracco G, et al. Helicobacter pylori infection and diabetes mellitus: the 2013 state of art. Panminerva Med 2013;55:277-81.

42 Furet J-P, Kong L-C, Tap J, et al. Differential adaptation of human gut microbiota to bariatric surgery-induced weight loss: links with metabolic and low-grade inflammation markers. Diabetes 2010;59:3049-57.

43 Moreno-Indias I, Sánchez-Alcoholado L, García-Fuentes E, et al. Insulin resistance is associated with specific gut microbiota in appendix samples from morbidly obese patients. Am J Transl Res 2016;8:5672-84.

44 Mouzaki M, Comelli EM, Arendt BM, et al. Intestinal microbiota in patients with nonalcoholic fatty liver disease. Hepatology 2013;58:120-7.

45 Roager HM, Licht TR, Poulsen SK, et al. Microbial enterotypes, inferred by the prevotella-to-bacteroides ratio, remained stable during a 6-month randomized controlled diet intervention with the new Nordic diet. Appl Environ Microbiol 2014;80:1142-9.

46 Fugmann M, Breier M, Rottenkolber M, et al. The stool microbiota of insulin resistant women with recent gestational diabetes, a high risk group for type 2 diabetes. Sci Rep 2015;5:13212.
47 Pedersen HK, Gudmundsdottir V, Nielsen HB, et al. Human gut microbes impact host serum metabolome and insulin sensitivity. Nature 2016;535:376-81.

48 Lin L, Wen Z-B, Lin D-J, et al. Correlations between microbial communities in stool and clinical indicators in patients with metabolic syndrome. World J Clin Cases 2018;6:54-63.

49 Murri M, Leiva I, Gomez-Zumaquero JM, et al. Gut microbiota in children with type 1 diabetes differs from that in healthy children: a case-control study. BMC Med 2013;11:46.

50 Leiva-Gea I, Sánchez-Alcoholado L, Martín-Tejedor B, et al. Gut microbiota differs in composition and functionality between children with type 1 diabetes and MODY2 and healthy control subjects: a case-control study. Diabetes Care 2018;41:2385-95.

51 Khorraminezhad L, Leclercq M, O'Connor S, et al. Dairy product intake modifies gut microbiota composition among hyperinsulinemic individuals. Eur J Nutr 2021;60:159-67.

52 Lv Y, Zhao X, Guo W, et al. The relationship between frequently used glucose-lowering agents and gut microbiota in type 2 diabetes mellitus. J Diabetes Res 2018;2018:1-7.

53 Gomez-Arango LF, Barrett HL, McIntyre HD, et al. Connections between the gut microbiome and metabolic hormones in early pregnancy in overweight and obese women. Diabetes 2016;65:2214-23.

54 Lambeth SM, Carson T, Lowe J, et al. Composition, diversity and abundance of gut microbiome in prediabetes and type 2 diabetes. $J$ Diabetes Obes 2015;2:1-7.

55 Takara Y, Endo H, Nakano R, et al. Smoking and drinking did not increase the failure of therapeutic Helicobacter pylori eradication by vonoprazan, clarithromycin, and amoxicillin. Digestion 2019;99:172-8. 